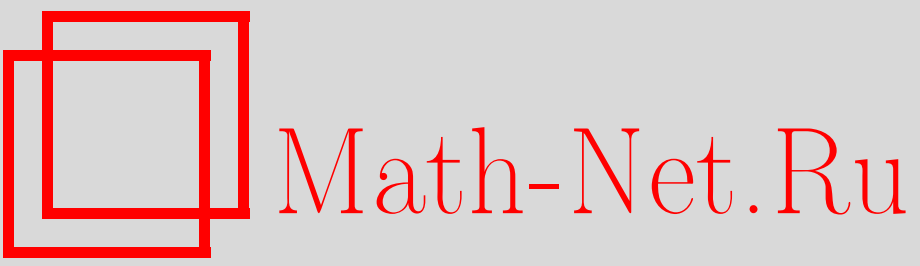

С. Т. Садэтов, Симплектическая структура на орбитах коприсоединенного представления - дифференциал рациональной 1-формы, УМH, 2005, том 60, выпуск $5,179-180$

DOI: https://doi.org/10.4213/rm1654

Использование Общероссийского математического портала Math-Net.Ru подразумевает, что вы прочитали и согласны с пользовательским соглашением

http://www. mathnet.ru/rus/agreement

Параметры загрузки:

IP : 52.87 .193 .239

26 апреля 2023 г., 13:37:57 


\title{
СИМПЛЕКТИЧЕСКАЯ СТРУКТУРА НА ОРБИТАХ КОПРИСОЕДИНЕННОГО ПРЕДСТАВЛЕНИЯ - ДИФ ФЕРЕНЦИАЛ РАЦИОНАЛЬНОЙ 1-ФОРМЫ
}

\author{
С. Т. САдЭТОВ
}

1. Пусть $\mathfrak{g}$ - конечномерная алгебра Ли над полем характеристики $0, \mathfrak{g}^{*}$ - сопряженное пространство. Для точки $\xi \in \mathfrak{g}^{*}$, элементов $\Xi, \Upsilon \in \mathfrak{g}$ и ковекторов $u=\operatorname{ad}_{\Xi}^{*} \xi, v=\operatorname{ad}_{\Upsilon}^{*} \xi$ определяется форма Кириллова-Березина-Костанта-Сурьо [1] $\omega_{\xi}(u, v)=\langle\xi,[\Xi, \Upsilon]\rangle$. На орбитах $O^{*}$ коприсоединенного представления группы Ли $G$ алгебры Ли $\mathfrak{g}$ она является симплектической структурой, см. [2; $§ 14.3],[3 ; \S 15.4]$. Многие уравнения механики гамильтоновы относительно нее. Пусть алгебра Ли $\mathfrak{g}$ - алгебраическая. Пусть $M \subseteq \mathfrak{g}^{*}$ - произвольное неприводимое $\mathrm{Ad}^{*}$-инвариантное алгебраическое подмногообразие. Следующее утверждение использовано автором в [4].

Теорема. На подмногообразии $M$ существует такая рациональная 1-форма $\mu$, что в ограничении на произвольную орбиту $O^{*} \subseteq M$ выполнено $\omega=d \mu$.

ПримеР. Если $x, y, z$ - ортонормированный относительно формы Киллинга базис алгебры Ли $s o(3, \mathbb{R})$ и $x=r \cos \theta \cos \varphi, y=r \cos \theta \sin \varphi, z=r \sin \theta$, где $r \geqslant 0,-\frac{\pi}{2} \leqslant \theta \leqslant \frac{\pi}{2}, 0 \leqslant \varphi \leqslant 2 \pi$, то $\omega=r \cos \theta d \theta \wedge d \varphi=d z \wedge d \operatorname{arctg} \frac{y}{x}$ - деленная на $r$ форма площади на стандартной сфере радиуса $r>0$ и может быть выбрано $\mu=r \sin \theta d \varphi=z \frac{x d y-y d x}{x^{2}+y^{2}}$.

Теорема и пример свидетельствуют в пользу того, что уравнения Эйлера-Пуанкаре на алгебрах Ли, см. [2], могут оказаться глобально лагранжевыми в подходящих координатах. Ср. с тем, что в [5] рассмотрена алгебра Ли $\mathbb{R}^{3} \dashv s o(3, \mathbb{R})$ и уравнения Кирхгофа на орбите коприсоединенного представления общего положения представлены в виде системы на двумерной сфере, лагранжевой с "монополем Дирака".

ДоКАЗАТЕЛЬСтво ПРИмЕРА. Присоединенное представление Ad сохраняет форму Киллинга. Ее уровень в $s o(3, \mathbb{R})^{*}$ - стандартная сфера $x^{2}+y^{2}+z^{2}=$ const. Если отождествить $s o(3, \mathbb{R})$ и $s o(3, \mathbb{R})^{*}$ с помощью формы Киллинга и взять $|\xi|=r$ и пару $u, v$ ортогональных единичных векторов из $T_{\xi} O^{*}$, то может быть выбрано $\Xi= \pm \frac{v}{r}, \Upsilon= \pm \frac{u}{r}$ для некоторой пары знаков. Тогда $\langle\xi,[\Xi, \Upsilon]\rangle= \pm r^{-2}\langle\xi,[v, u]\rangle= \pm r^{-1}$.

Не ограничивая общности, предположим, что $k$ - подполе поля коэффициентов $\mathbb{C}$, и заменим $\mathfrak{g}$ на $\mathfrak{g} \otimes_{k} \mathbb{C}$.

Доказательство теоремы основано на некоммутативной симплектической редукции, см. [6; дополнение $5 \mathrm{~A}],[2 ; \S 13.1-3],[3 ; 15.3]$. А именно, рассматривается соответствующая алгебре Ли $\mathfrak{g}$ связная алгебраическая группа $G$. Рассматриваются левое и правое действия группы $G$ на кокасательном расслоении $T^{*} G$ и их отображения моментов $\mu_{l}, \mu_{r}: T^{*} G \rightarrow \mathfrak{g}^{*}$, см. [6; дополнение 5], эквивариантные и пуассоновские, см. также $[2 ; \S 12.4]$. Из коммутативности действий легко следует, что орбита левого действия является уровнем отображения момента правого действия: $\mu_{r}=\xi$. Пусть $\mathrm{St}_{\xi} \subset G$ - стабилизатор точки $\xi$ при индуцированном представлении $\mathrm{Ad}^{*}$ группы $G$ на $\mathfrak{g}^{*}$. Фактормногообразие орбиты левого действия $\mu_{r}=\xi$ по правому действию подгруппы $\mathrm{St}_{\xi}$ является симплектическим и изоморфно орбите $O_{\xi}^{*}$ коприсоединенного представления группы $G$, проходящей через точку $\xi$, см. [2; $\S 14.3],[3 ; \S 15.4]$. Рассматривается расслоение орбиты левого действия группы по правому действию подгруппы и рациональное квазисечение $S$ этого расслоения, см. [7; $\S 2.5]$. Пусть $\nu$ и $\Omega$ - канонические 1-форма и симплектическая структура на $T^{*} G$. Ограничение условия $\Omega=d \nu$ на орбиту левого действия допускает дополнительное ограничение на квазисечение $S$. Поэтому ограничение $\omega$ на обиту $\left.O^{*} \omega\right|_{T O} O_{\text {является дифо- }}$ ференциалом алгебраической 1-формы - образа 1-формы $\nu$ при накрытии $S \rightarrow O^{*}$. Аддитивно усредняя алгебраическую 1-форму по ветвям над $O^{*}$, получаем рационалшную 1 -форму с тем же дифференциалом.

2. Доказательство теоремы. Пусть $\nu$ - каноническая 1 -форма на $T^{*} G$, см. [6; §37Б], порождающая каноническую симплектическую структуру $d \nu$. Тогда $\nu=\langle x, d g\rangle$, где $g \in G$, 
$x \in T_{g}^{*} G$, а $\langle\cdot, \cdot\rangle$ - спаривание $T_{g}^{*} G$ и $T_{g} G$. Пусть $L_{\xi}=\left\{b \in T^{*} G \mid \mu_{r}(b)=\xi\right\}$. Пусть $\eta$ - ковектор общего положения в $M, N \subset M$ - неприводимое алгебраическое подмногообразие, содержащее $\eta$, размерности, дополнительнй к размерности орбиты общего положения в $M$, трансверсальное орбите $O_{\eta}^{*}$. Пусть $S \subset \mu_{r}^{-1}(N)$ - неприводимое алгебраическое подмногообразие размерности, дополнительной к размерности орбиты правого действия подгруппы St $\eta$, трансверсальное некоторой такой орбите.

ПредЛОжениЕ 1. Регулярное отображение $\left.\mu_{l}\right|_{S}: S \rightarrow M$ определяет алгебраическое отображение $\left(\left.\mu_{l}\right|_{S}\right)^{-1}: M \rightarrow S$.

ПРЕДЛОЖЕНИЕ 2. ДЛя любого $\xi \in \mathfrak{g}^{*}$ выполнено $\mu_{l}\left(L_{\xi}\right)=O_{\xi}^{*}$.

Пусть $D$ - носитель дивизора ветвления и полюсов алгебраического отображения $\left(\left.\mu_{l}\right|_{S}\right)^{-1}$. Пусть точка $\xi \in M \backslash(D \cup \operatorname{sing} M)$ - общего положения, т.е. такая, что $\operatorname{dim} N \cap O_{\xi}^{*}=0$. Тогда $\bigcup_{\zeta \in N \cap O_{\xi}^{*}} L_{\zeta} \supset S \cap \bigcup_{\zeta \in O_{\xi}^{*}} L_{\zeta}=\left(\left.\mu_{l}\right|_{S}\right)^{-1}\left(O_{\xi}^{*}\right)$, где равенство вытекает из предложения 2 , так как объединение уровней $L_{\zeta}$, являющееся $\mu_{r}$-прообразом орбиты $O_{\xi}^{*}$, является $\mu_{l}$-прообразом орбиты $O_{\xi}^{*}$. Из сквозного вложения и редукции п. 1 следует первое равенство в следующей цепочке равенств, имеющей место в ограничении на орбиту $O_{\xi}^{*}$ :

$$
\omega=\left.(d \nu)\right|_{\zeta \in N \cap O_{\xi}^{*} T L_{\zeta}} \circ d\left[\left(\left.\mu_{l}\right|_{S}\right)^{-1}\right]=(d \nu) \circ d\left(\left.\mu_{l}\right|_{S}\right)^{-1}=d\left(\nu \circ\left(\left.\mu_{l}\right|_{S}\right)^{-1}\right) .
$$

Обозначим через $\Phi \subset \mathfrak{g}$ произволное линейное вложение факторпространства $\mathfrak{g} / \operatorname{ann}_{\mathfrak{g}} \eta$ в виде линейного подпространства, дополнительного к аннулятору $a_{\mathfrak{g}} \eta$. Рассмотрим семейство линейных отображений $\mathrm{ad}^{*} \zeta: \Phi \ni \Xi \rightarrow u \in T_{\zeta} O_{\zeta}^{*}$, регулярно зависящее от $\zeta \in M$ и для $\zeta$ общего положения являющееся изоморфизмом. Обращая это семейство, получаем семейство линейных отображений $\Xi(\cdot, \zeta): T_{\zeta} O_{\zeta}^{*} \rightarrow \Phi$, рационально зависящее от $\zeta \in M$. Следовательно, $\omega_{\xi}(u, v)=\langle\xi,[\Xi(u, \zeta), \Upsilon(v, \zeta)]\rangle-$ рациональная 2-форма на $M$.

Согласно [8], любое расширение вкладьвается в расширение Галуа (всех ветвей). Ветви 1-формы $\nu \circ\left(\left.\mu_{l}\right|_{S}\right)^{-1}$ на поверхности $M$ записываются в избыточных координатах на $M$ с коэффициентами из конечного расширения Галуа $F$ поля $\mathbb{C}(M)$ рациональных функций на $M$. На множестве ветвей 1-формы действует группа Галуа $\operatorname{Aut}(F / \mathbb{C}(M))$ расширения. По определению расширения Галуа, инварианты действия на $F$ - в точности элементы поля $\mathbb{C}(M)$. Усредняя сквозное равенство (1) по $\operatorname{Aut}(F / \mathbb{C}(M))$, получаем $\omega=d \mu$, где $\mu$ - требуемая рационалшая 1-форма.

Автор благодарит Э. Б. Винберга за внимательное прочтение рукописи и замечания по упрощению доказательства усреднения методом Галуа, Ф. Хирцебруха и А. В. Болсинова - за обсуждения. Автор благодарит Математический институт университета г. Бонна за гостеприимство и Немецкую службу академических обменов (DAAD) за стипендию A/04/06212, работа была также поддержана грантом НШ-1988.2003.1.

\section{СПИСОК ЛИТЕРАТУРЫ}

[1] Ф. А. Березин // Функц. анализ и его прил. 1967. Т. 1. № 2. С. 1-14. [2] J. E. Marsden, T.S. Ratiu. Introduction to Mechanics and Symmetry. New York: Springer-Verlag, 1999. [3] А.Т. Фоменко. Дифференциальная геометрия и топология. Дополнительные главы. М.: Изд-во Моск. ун-та, 1983. [4] С. Т. Садэтов. Гипотеза Гельфанда-Кириллова (1966) справедлива после конечных расширений тел Ли // Докл. РАН. 2005 (в печати). [5] С. П. Новиков // УМН. 1982. Т. 37. № 5. С. 3-49. [6] В. И. Арнольд. Математические методы классической механики. М.: Наука, 1989. [7] Э. Б. Винберг, В. Л. Попов // Итоги науки и техники. Современ. проблемы матем. Фундам. напр. Т. 55. М.: ВИНИТИ, 1989. С. 137-314. [8] И. Р. Шафаревич. Основы алгебраической геометрии. Т. 1, 2. М.: Наука, 1988.

Донской государственньй технический университет E-mail: sst@donpac.ru
Представлено Э. Б. Винбергом Принято редколлегией 22.07.2005 